\title{
The development of bone marrow adipose tissue
}

\author{
Jiejun Wang ${ }^{1}$ \\ ${ }^{1}$ Yangzhou High School, 18 Huaihai Road, Yangzhou, Jiangsu, China
}

\begin{abstract}
Adipose tissue, once viewed as a form of connective tissue and the storage of fat, has now been considered as an endocrine organ of energy homeostasis. Bone marrow is a vital organ in our body and takes up $4 \%$ of body mass. It is responsible for creating hematopoietic cells, marrow adipose tissue, and supportive stromal cells. Mesenchymal stem cells in bone marrow can differentiate to both osteoblasts and adipocytes. Studies show that the adipose tissue in the bone marrow has negative influence on bone development. And the differentiation of the adipocytes in bone marrow can be controlled by the regulation of PPARy, which is a member of the nucleus receptor family. In another word, the absence of PPAR $y$ in the bone marrow can be beneficial for bone development. Moreover, the osteogensis can also be regulated by $\mathrm{Cbf} \beta$ which is negatively correlated with adipogensis. In all kinds of adipose tissue, brown adipose tissue is distinct from others. It is triggered in cold conditions and release heat energy. Overexpression of PRDM16 in myoblasts can induce brown adipose.
\end{abstract}

\section{Introduction}

Adipose tissue in adult human body takes up approximately $18-25$ percentage which is a complex organ with profound effects on physiology and pathophysiology. Nowadays, the understanding of adipose tissue switches from a form of connective tissue to an endocrine organ of energy homeostasis. Most adipose tissue is white adipose tissue ( $~ 85 \%$ of total adipose tissue) which consisted of subcutaneous depots in the abdominal body parts. And brown adipose tissue and visceral adipose tissue is approximately $10 \%$. Bone marrow is a heterogeneous organ which has various alterations in composition with physiological changes and pathological states. Adipocytes are one of the most abundant cell types in bone marrow and have been found to influence bone remodeling by influencing bone cell differentiation and function.

\section{Development}

\subsection{The identification of adipose tissue}

Bone marrow adipose tissue development differs from that of extramedullary adipose tissues. It is not correlated to usual adiposity parameters like waist-to -hip ratio or body mass [1-3] and its formation seems to be independent from white adipose tissue in human body. BMAT can be considered a vital indication of bone integrity as it, for example, increases sharply in the situation of anorexia nervosa caused bone loss. Also, when conducting a calorie-restricted diet, other extramedullary fat tissues decrease in amount while no significant change is shown in BMAT content. [4]

The subpopulations of cells in bone marrow are committed to osteoblast or adipocyte lineage [5]. The bone marrow contains hematopoietic stem cells and mesenchymal stem cells (MSC). MSC are plasticadherent, clonal cells that are progenitors for osteoblasts and adipocytes. Fig. 1 shows that MSCs can differentiate into other cell types.

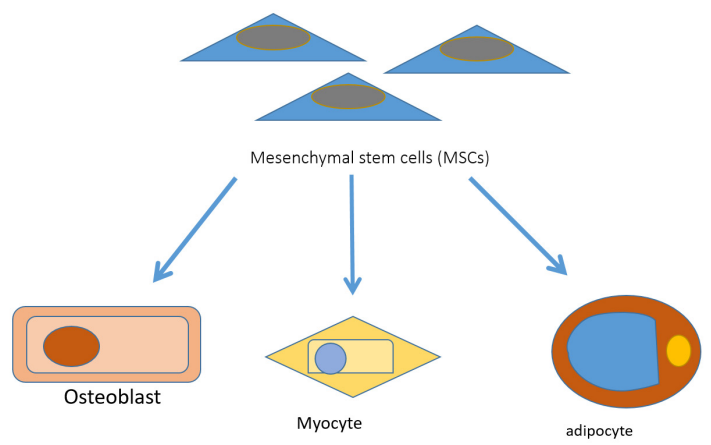

Figure 1. MSCs differentiate into other cell types.

To test the distinct progenitor cell populations for adipocytes and osteoblasts, Clonal MSC are derived from murine bone marrow and characterized as mMSC1 and mMSC2. Both cell lines are positive for the murine MSC marker Sca-1 while mMSC1 was also positive for CD13. MSC1 can differentiate into adipocytes evidenced by expressing adipocyte markers and visualized by Oil Redd O staining. MSC2 can differentiate into osteoblasts by up regulation of osteoblastic markers and formation of alizarin red. 
Experiments prove that treatment with thiazolidinediones (TZDs) and high-fat feeding increase the circulation of BM-derived progenitor cells to adipose tissue and their differentiation into a novel adipocyte population [6]. It is believed that new adipocytes are risen from the resident adipose tissue pre-adipocytes and mesenchymal progenitor cells, the progenitor cells form bone marrow can also contribute to formation of new adipocytes in adipose tissue. To test this hypothesis, they transplant whole BM-derived cell from GFP transgenic mice into wild-type mice under high fat diet or rosiglitazone (which is a PPAR $\gamma$ agonist)-treated. It turns out that the number of $\mathrm{GFP}^{+}$multilocular adipocytes was significantly increased in these mice. What's more, these ML adipocytes expressed adiponectin, perilipin, fatty acid-binding protein (FABP), leptin, C/EBP $\beta$, and PPAR $\gamma$ and they also showed increased mitochondrial content. The result shows the new adipose tissue does differentiate from the resident BM-derived progenitors.

\subsection{The role of transcription factors in osteogenesis}

PPAR $\gamma$ is a member of nuclear receptor family. PPAR $\gamma 1$ and PPAR $\gamma 2$ are different PPAR $\gamma$ isoforms in fat and PPAR $\gamma 2$ has been identified as a key regulator of two fat cell enhancers and its transcriptional activation is stimulated by lipids or lipid-like compounds. PPAR 2 is expressed at higher level and is induced at the early stage of cultured adipocyte cell lines differentiation than PPAR $\gamma 1$. Overexpression of PPAR $\gamma 2$ promotes cultured fibroblast cells adipogenesis in a PPAR $\gamma$ activatordependent manner. [7] The adipogenesis also can be induced by overexpressing PPAR $\gamma 2$ and C/EBP $\beta$ together in the absence of PPAR $\gamma$ activator. The insufficiency of PPAR $\gamma$ enhance bone formation by promoting osteoblastogenesis from bone marrow progenitor cells [8]. Homozygous PPAR $\gamma$-deficient ES cells cannot differentiate into adipocytes, but can differentiate into osteoblasts, and these were restored by reintroduction of the PPAR $\gamma$ gene. Heterozygous PPAR $\gamma$-deficient mice exhibited high bone mass with increased osteoblastogenesis, but normal osteoblast and osteoclast functions, and this effect was not mediated by insulin or leptin. This suggests that the absence of PPAR $\gamma$ benefits osteogensis.

$\mathrm{Cbf} \beta$ is commonly described as a key transcription factor in osteogenesis, it negatively correlates to adipogenesis in bone marrow [9]. To study the effect of transcription factor $\mathrm{Cbf} \beta$ on osteoblast lineage commitment, this factor was deleted in different stages of osteoblast lineage using $\mathrm{Cbf} \beta \mathrm{f} / \mathrm{fPrx} 1-\mathrm{Cre}, \mathrm{Cbf} / \mathrm{f} / \mathrm{fCol} 2$ $\alpha 1-\mathrm{Cre}$, and Cbf $\beta f / f O s x-C r e$ mice. The Cbf $\beta f / f P r x 1-C r e$, $\mathrm{Cbf} \beta \mathrm{f} / \mathrm{fCol} 2 \alpha 1-\mathrm{Cre}$, and $\mathrm{Cbf} \beta \mathrm{f} / \mathrm{fOsx}-\mathrm{Cre}$ mice exhibited severe osteoporosis with substantial accumulation of marrow adipocytes resembling aged bone from enhanced adipogenesis. Deletion of $\operatorname{Cbf} \beta$ in calvarial cells and bone marrow mesenchymal stem cells stimulate the cells adipogenesis and inhibit osteogenesis via activating
Wnt10b/ $\beta$-catenin signaling and inhibiting $(\mathrm{C} / \mathrm{EBP} \beta)$ expression.

Among all the fat tissues, another differs from the others being the being brown adipose tissue (WAT). In order to figure out the effect of PRDM16 on regulation, they used the Myf5-Cre: R26R3-YFP mice [10]. YFP was only detected in skeletal muscle and BAT but not in WAT. Thus, brown fat and skeletal muscle arise from Myf5- expressing progenitors. PRDM16 is a transcriptional regulator that can control a switch between skeletal myoblasts and brown adipocytes. Deletion of PRDM16 from primary brown adipocytes induces muscle differentiation. And overexpression of PRDM16 in myoblasts stimulates their differentiation into brown adipocytes. PRDM16 promotes brown adipocyte differentiation by binding to PPAR $\gamma$ and activating its transcriptional function. Ectopic expression of PRDM16 and PPAR $\gamma 2$ can convert myogenic cells into brown adipocytes. Based on the study in this article, it is worth investigating the possibility of prdm16 functions in bone marrow and how it works.

\section{Summary}

Bone marrow adipocytes have characteristic of adipocytes but it differs from normal extramedullary fat tissue. Bone marrow adipocytes are also important for critical physiological process of bone as well as the relevance of these cells to bone loss disorders.

\section{References}

1. Justesen, J., Stenderup, K., Ebbesen, E. N., Mosekilde, L., Steiniche, T., \& Kassem, M. Biogerontology 2, 165-171 (2001)

2. Shen, W., Chen, J., Punyanitya, M., Shapses, S., Heshka, S., \& Heymsfield, S. B. Osteoporosis international 18, 641-647 (2007)

3. Di Iorgi, N., Rosol, M., Mittelman, S. D., \& Gilsanz, V. The Journal of Clinical Endocrinology \& Metabolism, 93, 2281-2286 (2008)

4. Cordes, C., Dieckmeyer, M., Ott, B., Shen, J., Ruschke, S., Settles, M., ... \& Skurk, T. Journal of Magnetic Resonance Imaging, 42, 1272-1280 (2015)

5. Post, S., Abdallah, B. M., Bentzon, J. F., \& Kassem, M. Bone, 43, 32-39 (2008)

6. Crossno, J. T., Majka, S. M., Grazia, T., Gill, R. G., \& Klemm, D. J. The Journal of clinical investigation, 116, 3220-3228 (2006)

7. Tontonoz, P., Hu, E., \& Spiegelman, B. M. Cell, 79, 1147-1156 (1994)

8. Akune, T., Ohba, S., Kamekura, S., Yamaguchi, M., Chung, U. I., Kubota, N., ... \& Kadowaki, T. The Journal of clinical investigation, 113, 846-855 (2004)

9. Wu, M., Wang, Y., Shao, J. Z., Wang, J., Chen, W., \& Li, Y. P. Proceedings of the National Academy of Sciences, 114, 10119-10124 (2017) 
10. Seale, P., Bjork, B., Yang, W., Kajimura, S., Chin, S., Kuang, S., ... \& Tempst, P. Nature, 454(7207), 961 (2008) 